\title{
Anesthesiologist's hand hygiene and disinfection of reusable rubber tourniquet with alcohol swabs before intravascular cannulation
}

\author{
Ji Yeon Kim, Hyun-Joo Ahn, Eun-Kyung Lee, and Hyun Byung Chae \\ Department of Anesthesiology and Pain Medicine, Samsung Medical Center, Sungkyunkwan University School of Medicine, Seoul, \\ Korea
}

Patients undergoing surgery have an increased risk for hospital-acquired infections. Various causes such as the catabolic response to surgery, old age, and co-existing diseases such as diabetes mellitus, make these patients more susceptible to nosocomial infections.

One of the major efforts to prevent hospital-acquired infections is personal hygiene of the health care provider, which reportedly reduces the incidence of hospital-acquired infections [1]. Another effort is the sterilization of instruments used in the operating rooms. Previous studies identified the anesthesiologists' hands and anesthetic equipment as possible contributing factors to the transmission of pathogenic organisms, as they are frequently in contact with upper airway secretions and the blood of patients [2].

We conducted this study with the infection control team of our hospital to assess the hygiene status of re-usable rubber tourniquets and to determine their proper care in the operating room. Reusable tourniquets are in direct contact with multiple surgical patients and the anesthesiologist's hands. We tried to determine whether simple alcohol swabbings could disinfect re-usable tourniquets.

This study took place in 30 operating rooms of 2 hospital buildings. The operating rooms were used for a wide range of surgical specialties. Collection and inoculation was performed in each operating room with aseptic technique. First, we analysed the gross appearance of the tourniquet to determine whether it was visibly soiled with dirt or blood. Second, the author who wore sterile gloves cut the tourniquet into 2 equal sizes using sterile scissors. Third, one half was rolled and pressed onto blood agar plates (BAP) and the other half was twice swabbed with $83 \%$ ethyl-alcohol, dried, and then rolled and pressed onto BAP. Subsequently, each part of the divided tourniquet was immediately immersed in a conical tube containing $40 \mathrm{ml}$ of distilled water.

We used 2 methods to determine the hygiene of tourniquets, i.e. rolling and pressing onto BAP and inoculation of tourniquet derived solution into TSA.

BAP, which were inoculated with each divided tourniquet, were incubated at $35^{\circ} \mathrm{C}$ for 48 hours. Organisms were identified from colony morphology, coagulase test, catalase test, and pyruvate reaction test by an experienced biomedical scientist. Colonies presumptively identified as Staphylococcus aureus or Enterococcus were subjected to further tests for antibiotic susceptibility to identify methicillin-resistant S. aureus (MRSA) or vancomycin-resistant Enterococcus (VRE).

The TSA media was incubated at $35^{\circ} \mathrm{C}$ for 48 hours and the colonies were counted after one week. The total colony count was determined in colony-forming units (CFUs)/ml.

The questionnaire was distributed to anesthesiologists including professors, residents, and nursing staff involved with the peripheral intravascular catheter insertion in the operating room, to determine personal hygiene, hand washing or use of

Corresponding author: Hyun-Joo Ahn, M.D., Ph.D., Department of Anesthesiology and Pain Medicine, Samsung Medical Center, Sungkyunkwan University School of Medicine, 81, Irwon-ro, Gangnam-gu, Seoul 135-710, Korea. Tel: 82-2-3410-2470, Fax: 82-2-3410-0361, E-mail: hyunjooahn@skku.edu

(c) This is an open-access article distributed under the terms of the Creative Commons Attribution Non-Commercial License (http:// creativecommons.org/licenses/by-nc/3.0/), which permits unrestricted non-commercial use, distribution, and reproduction in any medium, provided the original work is properly cited. 
Table 1. Hand Hygiene Habits Regarding Tourniquet Use $(n=62)$

\begin{tabular}{cccc}
\hline \multirow{2}{*}{ Hand hygiene } & Pre- cannulation & During cannulation & After-cannulation \\
\cline { 2 - 4 } & Soap or alcohol gel & Wearing gloves & Soap or alcohol gel \\
\hline Always & $37.1 \%$ & $4.9 \%$ & $61.3 \%$ \\
Sometimes & $43.5 \%$ & $27.4 \%$ & $37.1 \%$ \\
Never & $19.4 \%$ & $67.7 \%$ & $1.6 \%$ \\
\hline
\end{tabular}

antimicrobial hand rubs, when the tourniquet was applied to the surgical patients in the operating room.

Paired t-test was used to compare the count of CFUs before and after alcohol swabbings. SAS software version 9.3 (SAS Institute Inc., Cary, NC, USA) was used for these analyses.

The expiration dates of the tourniquets were unknown. The most common reason for replacing a tourniquet was loss of the old one. Fifteen of the 30 (50\%) tourniquets in the study were visibly soiled in appearance. Tourniquets from orthopedic operations showed the highest bacterial contamination (44.75 CFUs/ $\mathrm{ml})$.

We evaluated bacterial contamination of the re-usable tourniquets both before and after alcohol swabbing. No colonies were grown on blood agar plate culture in the alcohol swab - intervention group (100\%). However various colonies were grown in the non-intervention group (100\%) and they were identified by a microbiologist. Most colonies were S. aureus or enterococcus, however, none were positive for MRSA or VRE. All tourniquets before alcohol swabbings showed positive bacterial growth in the TSA culture. The amount of organisms found on the tourniquets (CFUs/ml) decreased significantly after alcohol swabbings (mean \pm SD: $24.5 \pm 6.3$ vs. $3.5 \pm 0.89$, before and after swabbings respectively, $\mathrm{P}=0.001$ ). Alcohol swabbing ( $83 \%$ ethyl-alcohol) twice before the use of tourniquet decreased bacterial contamination considerably (mean reduction: $90.2 \pm 11.5 \%$ ).

Among the anesthesia staff who answered the questionnaire, $37 \%$ always washed their hands with soap or alcohol gel before intravascular catheter insertion, and $44 \%$ of the staff occasionally did. However, $19 \%$ of the staff made no attempts to clean their hands before cannulation (Table 1).

The aim of this study was to confirm bacterial contamination of re-usable tourniquets in the operating room and to determine whether twice alcohol swabbings could eliminate bacterial organisms on the tourniquet.

All collected tourniquets in our study were negative for MRSA and VRE. However, previous studies reported differently. One study reported that $36 \%$ of tourniquets were positive for S. aureus and 12\% were MRSA-positive [3]. Another study revealed that MRSA was isolated from $24.4 \%$ of collected tourniquets [4]. Results from our study differed from previous studies likely since the prior studies were conducted in general wards rather than in the operating rooms, which have relatively aseptic conditions.

Single use tourniquet is ideal, however as long as non-disposable tourniquets are used for various reasons, proper infection control is required. Kerstein and Fellowes [5] recommended disposable tourniquets because reducing transmission to patients by single-use tourniquets is more cost-effective, considering the cost of hospital-acquired infections.

In conclusion, tourniquet contamination is dependent on the hospital personnel's hygiene and that of the entire hospital. Poor hygiene combined with the careless use of tourniquets make reusable tourniquets a vehicle of hospital-acquired infections.

\section{References}

1. Eaton L. Hand washing is more important than cleaner wards in controlling MRSA. BMJ 2005; $330: 922$.

2. Baillie JK, Sultan P, Graveling E, Forrest C, Lafong C. Contamination of anaesthetic machines with pathogenic organisms. Anaesthesia 2007; 62: 1257-61.

3. Elhassan HA, Dixon T. MRSA contaminated venepuncture tourniquets in clinical practice. Postgrad Med J 2012 ; 88: $194-7$.

4. Leitch A, McCormick I, Gunn I, Gillespie T. Reducing the potential for phlebotomy tourniquets to act as a reservoir for methicillin-resistant Staphylococcus aureus. J Hosp Infect 2006; 63: 428-31.

5. Kerstein RL, Fellowes C. Novel fit for purpose single use tourniquet: best of both worlds. J Med Eng Technol 2009; 33: 475-80. 